\title{
Meccanismo d'azione dei farmaci più recenti, teriflunomide e alemtuzumab e implicazioni terapeutiche
}

\section{Francesco Patti}

Dipartimento "GF Ingrassia, Scienze mediche e chirurgiche e tecnologie avanzate", Sezione Neuroscienze, Centro Sclerosi Multipla, Università di Catania

\section{Introduzione}

Nell'ultimo ventennio, la gestione della sclerosi multipla $(\mathrm{SM})$ è divenuta sempre più complessa per la disponibilità di numerosi farmaci modificanti il decorso della malattia. Si dispone oggi di 11 molecole approvate con vari gradi di efficacia, specifici profili di sicurezza, specifici meccanismi di azione. Fino al 2011, si disponeva di farmaci iniettivi per via intramuscolare (i.m.) o sottocutanea (s.c.) [interferoni e copolimero] o per via venosa (e.v.) [natalizumab]. Nel 2011 e nel 2014 sono stati approvati i farmaci orali fingolimod, teriflunomide e BG-12 (dimetilfuramato), e ancora più recentemente alemtuzumab (altro farmaco e.v.). Altri farmaci sono in fase di svi-

\section{Indirizzo per la corrispondenza:}

Francesco Patti

e-mail: patti@unict.it

Accettato: 16/10/2015 - Pubblicato online: 26/11/2015

(C) 2015 The Authors. This article is published by HPS Srl and licensed under Creative Commons Attribution-NC-ND 4.0 International (CC BY-NC-ND 4.0). Any commercial use is not permitted and is subject to Publisher's permissions. Full information is available at www.aboutpharma.com/publishing/riviste/aboutopen/ luppo sia per la forma a recidive e remissioni sia per la forma progressiva. Di seguito vengono riportate le principali evidenze cliniche relative a due di queste nuove molecole, alemtuzumab e teriflunomide.

\section{Alemtuzumab}

Alemtuzumab (IgG1) è un anticorpo monoclonale umanizzato diretto contro l'antigene CD52 espresso sulla superficie di linfociti maturi, monociti, cellule dendritiche e granulociti [1]. L'azione di alemtuzumab si esplica con la morte delle cellule per citolisi complemento-dipendente e apoptosi. La somministrazione di alemtuzumab determina rapida e prolungata linfocitopenia e conseguente ricostituzione linfocitaria radicalmente riformata nella compagine dei Treg e delle cellule T autoreattive [2]. Il 17 settembre del 2013, Genzyme ha annunciato che EMA aveva autorizzato alemtuzumab per il trattamento della SM. In seguito alemtuzumab è stato approvato in diverse parti nel mondo e, dopo un primo rifiuto, la Food and Drug Administration (FDA) statunitense ha accettato di riconsiderare la sottomissione 
degli studi registrativi prodotti da Genzyme. I primi studi hanno dimostrato che esiste una finestra terapeutica - un tempo - all'interno del quale alemtuzumab è in grado di spegnere l'infiammazione della malattia. Di contro, gli stessi studi hanno dimostrato che nei pazienti in fase di progressione, alemtuzumab risulta largamente inefficace [3-5]. Un ulteriore studio di fase II ha dimostrato che alemtuzumab, somministrato sia a 12 che a $24 \mathrm{mg}$ per 5 giorni, è più efficace di interferone beta-1a alla dose di 44 $\mu g$, somministrato 3 giorni la settimana, in termini di minore tasso annualizzato di recidive, riduzione della progressione della disabilità, riduzione del carico lesionale in $\mathrm{T} 2$ e riduzione del carico lesionale gadolinio-positivo [6]. Sottoanalisi di questo studio hanno dimostrato che il ricostituito repertorio di linfociti circolanti produceva fattori neurotrofici in grado di promuovere la sopravvivenza neuronale, la crescita assonale, nonché la maturazione e la sopravvivenza degli oligodendrociti [7]. Questi risultati sono stati quasi del tutto confermati dagli studi registrativi di fase III, CARE MS I e CARE MS II, che hanno confrontato alemtuzumab somministrato alla dose di $12 \mathrm{mg}$ per 5 giorni consecutivi e altri 3 giorni consecutivi dopo 1 anno e interferone beta-1a 44 $\mu \mathrm{g}$ tre volte la settimana $[8,9]$. Uno studio recente "real life" (periodo di osservazione 7 anni) ha dimostrato una stabilizzazione della malattia in circa il $70 \%$ dei pazienti [10].

Alemtuzumab ha un basso profilo di sicurezza; gli eventi avversi più frequentemente riscontrati sono le infezioni e una serie di malattie autoimmuni, senza considerare gli eventi legati all'immediata liberazione di citochine. Porpora trombocitopenica, tiroidite autoimmune, sindrome di Goodpasture e infezioni erpetiche possono limitarne l'uso. Le manifestazioni autoimmunitarie possono presentarsi anche alcuni mesi dopo la somministrazione di alemtuzumab; il rischio di infezioni è più strettamente connesso al tempo della sua somministrazione. Tuttavia il trattamento con farmaci antivirali e un buon programma di accurata vigilanza medica possono ridurre al minimo i "safety concerns". Nonostante il rischio di malattie autoimmuni, alemtuzumab può essere un ideale candidato per i pazienti con SM particolarmente attiva. Grazie alla sua efficacia, alemtuzumab potrebbe essere impiegato anche come farmaco di induzione, per i primi 2 anni, sequenzialmente associato a un farmaco di prima linea (interferoni, copolimero, teriflunomide), di solito gestibile per un lungo periodo. A oggi non sono stati riportati casi di leucoencefalopatia multifocale progressiva in pazienti con SM trattati con alemtuzumab [11]. Migliorando le competenze dei neurologi e la loro fiducia nella gestione dei rischi connessi con alemtuzumab, si potrebbe pensare anche a un possibile impiego di questo farmaco in prima linea, per quei pazienti più giovani con maggiori aspetti di infiammazione [12] (Figura 1).

\section{Teriflunomide}

Teriflunomide, metabolita attivo di leflunomide, farmaco in uso nel trattamento dell'artrite reumatoide, è stato approvato per il trattamento della sclerosi multipla recidivante remittente (SMRR) dalla FDA il 13 settembre 2012 e dall'Agenzia Europea per i Medicinali (EMA) il 26 agosto 2013. Teriflunomide inibisce selettivamente e reversibilmente la diidroorotato deidrogenasi, enzima mitocondriale necessario per la sintesi de novo delle pirimidine. Grazie a questa azione si riduce la quota di linfociti $\mathrm{T}$ e $\mathrm{B}$ attivati, in grado di migrare e attraversare la barriera ematoencefalica, espletando un effetto citostatico [14]. Pertanto il farmaco può essere definito "immunomodulante".

Teriflunomide è stata studiata come farmaco da usare in monoterapia o in add on [15]. I primi studi di fase II hanno dimostrato sensibile e significativa efficacia delle due dosi testate (7 e 14 $\mathrm{mg} /$ die) sia sul numero di lesioni captanti gadolinio sia sul numero di nuove lesioni o di altre che aumentavano di volume nelle sequenze T2 pesate [16]. I successivi studi di fase III, TEMSO e TOWER, hanno dimostrato che i pazienti 


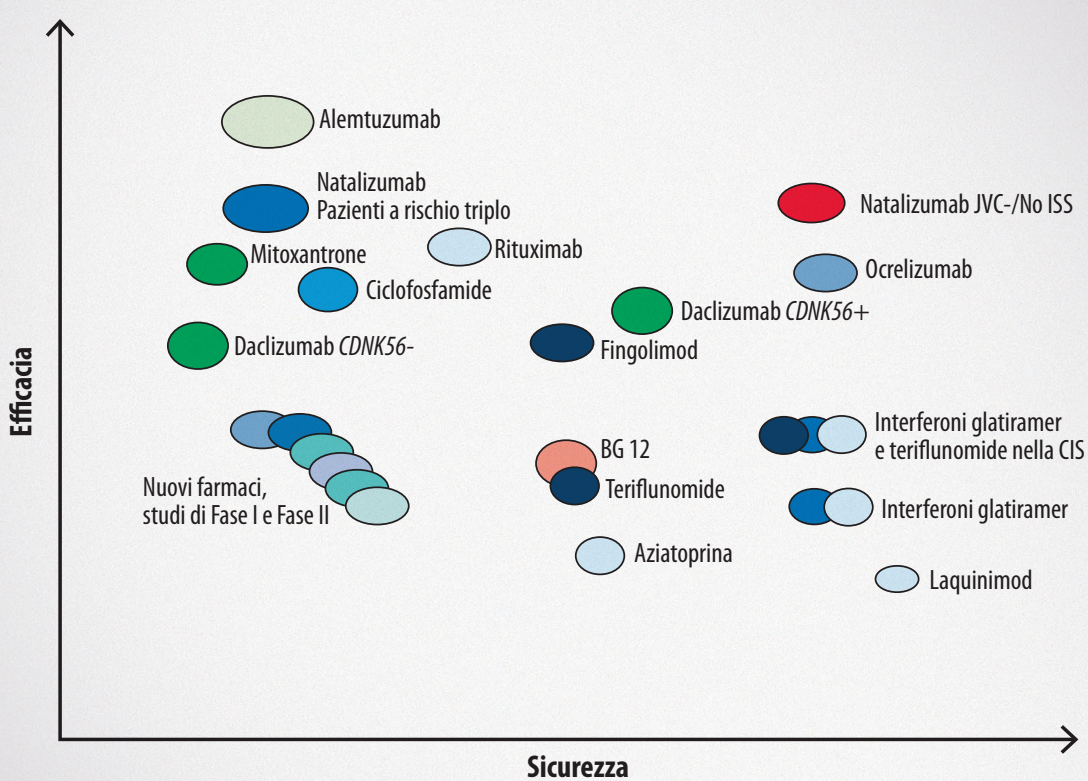

Figura 1. Sicurezza ed efficacia di specifiche terapie nella sclerosi multipla (modificata graficamente da [13]). CIS, sindrome clinicamente isolata; No ISS, no immunosoppressori.

trattati con la dose più alta, $14 \mathrm{mg}$, miglioravano in termini di tasso annualizzato di recidive, progressione della disabilità misurata con la Expanded Disability Status Scale (EDSS), confermando quanto rilevato negli studi di fase II sui parametri di risonanza magnetica (RM) [17-19]. Due successivi studi di estensione a 5 e 9 anni hanno dimostrato che la gran parte dei pazienti rimaneva in trattamento con teriflunomide con relativa stabilità dell'EDSS [20]. In un altro studio di fase III, denominato TENERE, non è stata riscontrata alcuna differenza in termini di efficacia clinica fra il gruppo di pazienti trattati con alta dose di teriflunomide e il gruppo trattato con interferone beta-1a $44 \mu \mathrm{g}$ (Figura 2) [21]. È stato inoltre dimostrato che teriflunomide aveva ridotto il rischio di recidiva o di una nuova lesione alla RM rispetto al placebo a entrambe le dosi testate in un'ampia coorte di pazienti affetti da sindrome clinicamente isolata (CIS) di malattia demielinizzante, suggerendone l'uso precoce [18]. Una serie di altri studi hanno infine dimostrato che teriflunomide, usata in combinazione con altri farmaci (interferone, glatiramer), è più efficace di glatiramer o di interferone impiegati da soli [22]. È attualmente in corso uno studio (TERIKIDS) sulla possibile somministrazione di teriflunomide in pazienti pediatrici con SMRR. L'uso di teriflunomide si è rivelato sicuro e il farmaco è ben tollerato. Gli eventi avversi riscontrati sono stati prevalentemente di grado moderato; tra i più frequenti si annoverano l'assottigliamento dei capelli, nausea, diarrea e aumento delle transaminasi; sono stati inoltre segnalati alcuni casi di neuropatia. L'incidenza di eventi avversi gravi è risultata sovrapponibile a quella del gruppo placebo [23]. Nonostante l'FDA attribuisca un rischio di classe $\mathrm{X}$ per la categoria "gravidanze" per il possibile rischio teratogeno di teriflunomide, va sottolineato che questo effetto è stato dimostrato unicamente negli animali da esperimento [24]. Teriflunomide, il secondo farmaco orale approvato per la cura della SM, ha un profilo di efficacia simile a quello di interferoni e copolimero. Può essere inoltre impiegata in quei pazienti che hanno scarsa compliance all'uso prolungato di farmaci iniettivi. 


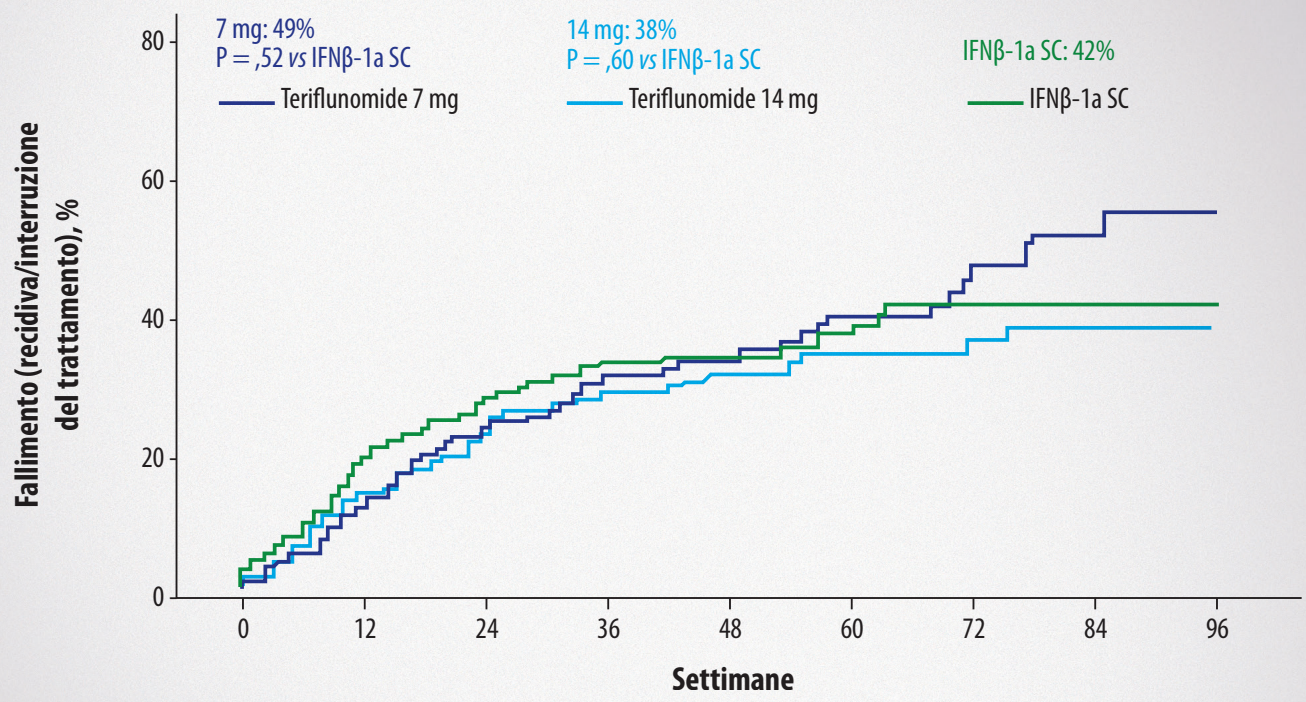

Figura 2. Assenza di differenze significative nell'efficacia clinica di teriflunomide 14 mg vs interferone beta-1a (IFNB-1a) [modificata graficamente da [21]]. SC, sottocute.

\section{Conclusioni}

I due nuovi farmaci qui delineati hanno diverso meccanismo d'azione, diverso profilo di sicurezza e diversa efficacia. Se si vuole classificarli, teriflunomide appartiene alla serie dei farmaci di prima linea e alemtuzumab alla serie di seconda o terza linea. Tuttavia, il diverso meccanismo d'azione dei due farmaci rende il lavoro dei neurologi più stimolante, teso alla ricerca del farmaco adatto per ciascun paziente nelle diverse fasi di malattia.

In quest'ottica teriflunomide potrebbe essere considerata farmaco di prima scelta o farmaco alternativo per quei pazienti che non tollerano più la via iniettiva propria degli interferoni e del copolimero o la sindrome simil-influenzale associata agli interferoni. Teriflunomide potrebbe essere utilizzata in add on con altre molecole di prima linea o come farmaco-ponte prima di passare al trattamento con agenti più potenti (natalizumab, alemtuzumab) o per consentire il passaggio da un farmaco ritenuto più potente (fingolimod, natalizumab) verso un altro agente, percepito come ulteriormente più efficace (alemtuzumab).
Alemtuzumab è stato approvato dopo la dimostrazione di superiorità nei confronti di interferone beta-1a alla dose di $44 \mu \mathrm{g}$. Da molti ritenuto farmaco di terza linea, e persino più efficace di natalizumab, ha mostrato un profilo di efficacia di notevole impatto per tutti gli aspetti della SM guidati dalla fase infiammatoria di malattia. Tuttavia, la sua tossicità (immunosoppressione prolungata), l'alto tasso di autoimmunità secondaria e il rischio di aumento di infezioni e neoplasie possono limitarne l'uso futuro. Va sottolineato che al momento costituisce il farmaco di elezione per quei pazienti con malattia in fase attiva che hanno fallito una o due linee di terapie specifiche e per quei pazienti che presentino sin dall'inizio un particolare profilo di attività di malattia (elevato carico lesionale, lesioni gadolinio-captanti, lesioni spinali alla RM) e un elevato tasso di recidive.

In ogni caso, è necessario che i Neurologi conoscano la storia clinica e terapeutica dei propri pazienti e sappiano prevenire e contrastare gli eventuali effetti collaterali connessi con le nuove terapie con un'attenta, vigile e frequente azione di monitoraggio clinico. 


\section{Disclosures}

Conflicts of interest: The author declares he received personal compensation for speaking activities and advisory board activities by Almiral, Bayer, Biogen, Merck, Sanofi Genzyme, Novartis and Teva.

\section{Bibliografia}

1. Alinari L, Lapalombella R, Andritsos L, et al. Alemtuzumab (Campath-1H) in the treatment of chronic lymphocytic leukemia. Oncogene. 2007;26:3644-53.

2. Coles AJ. Alemtuzumab therapy for multiple sclerosis. Neurotherapeutics. 2013;10(1):29-33.

3. Moreau T, Thorpe J, Miller D, et al. Preliminary evidence from magnetic resonance imaging for reduction in disease activity after lymphocyte depletion in multiple sclerosis. Lancet. 1994;344(8918):298-301.

4. Coles AJ, Wing MG, Molyneux P, et al. Monoclonal antibody treatment exposes three mechanisms underlying the clinical course of multiple sclerosis. Ann Neurol. 1999;46(3):296-304.

5. Coles AJ, Cox A, Le Page E, et al. The window of therapeutic opportunity in multiple sclerosis: evidence from monoclonal antibody therapy. J Neurol. 2006;253(1):98-108.

6. Coles AJ, Fox E, Vladic A, et al. Alemtuzumab versus interferon beta-1a in early relapsing-remitting multiple sclerosis: post-hoc and subset analyses of clinical efficacy outcomes. Lancet Neurol. 2011; 10(4):338-48.

7. Jones JL, Anderson JM, Phuah CL, et al. Improvement in disability after alemtuzumab treatment of multiple sclerosis is associated with neuroprotective autoimmunity. Brain. 2010;133(Pt 8):2232-47.

8. Cohen JA, Coles AJ, Arnold DL, et al. Alemtuzumab versus interferon beta $1 \mathrm{a}$ as first-line treatment for patients with relapsing-remitting multiple sclerosis: a randomised controlled phase 3 trial. Lancet. 2012;380(9856):1819-28.

9. Coles AJ, Twyman CL, Arnold DL, et al. Alemtuzumab for patients with relapsing multiple sclerosis after disease-modifying therapy: a randomised controlled phase 3 trial. Lancet 2012;380(9856):1829-39.

10. Tuohy O, Costelloe L, Hill-Cawthorne G, et al. Alemtuzumab treatment of multiple sclerosis: long-term safety and efficacy. J Neurol Neurosurg Psychiatry. 2014;86(2):208-15.

11. Garnock-Jones KP. Alemtuzumab: a review of its use in patients with relapsing multiple sclerosis. Drugs. 2014;74(4):489-504.
12. D’Amico E, Leone C, Caserta C, Patti F. Oral drugs in multiple sclerosis therapy: an overview and a critical appraisal. Exp Rev Neurother. 2015;15(7):803-24.

13. D'Amico E, Messina S, Caserta C, Patti F. A critical appraisal of daclizumab use as emerging therapy. Exp Opin Drug Saf. 2015;14(7):1157-68.

14. Bar-Or A, Pachner A, Menguy-Vacheron F, et al. Teriflunomide and its mechanism of action in multiple sclerosis. Drugs. 2014;74(6):659-74.

15. Garnock-Jones KP. Teriflunomide: a review of its use in relapsing multiple sclerosis. CNS Drugs. 2013;27(12):1103-23.

16. O'Connor P, Li D, Freedman M, et al. A phase II study of the safety and efficacy of teriflunomide in multiple sclerosis with relapses. Neurology. 2006;66:894-900.

17. O'Connor P, Wolinsky J, Confavreux C, et al. Randomized trial of oral teriflunomide for relapsing multiple sclerosis. N Engl J Med. 2011;365:1293-303

18. Miller AE, Wolinsky JS, Kappos L, et al. Oral teriflunomide for patients with a first clinical episode suggestive of multiple sclerosis (TOPIC): a randomised, double-blind, placebo-controlled, phase 3 trial. Lancet Neurol. 2014;13(10):977-86.

19. Confavreux C, O'Connor P, Comi G, et al. Oral teriflunomide for patients with relapsing multiple sclerosis (TOWER): a randomised, double-blind, placebo-controlled, phase 3 trial. Lancet Neurol. 2014;13(3):247-56.

20. Confavreux C, Li D, Freedman M, et al. Long-term follow-up of a phase 2 study of oral teriflunomide in relapsing multiple sclerosis: safety and efficacy results up to 8.5 years. Mult Scler. 2012;18:1278-89

21. Vermersch P, Czlonkowska A, Grimaldi L, et al. Evaluation of patient satisfaction from the TENERE study: a comparison of teriflunomide and subcutaneous interferon beta-1a in patients with relapsing multiple sclerosis. J Neurol. 2012;259(Suppl 1):1-236.

22. D'Amico E, Caserta C, Patti F. Monoclonal antibody therapy in multiple sclerosis: critical appraisal and new perspectives. Exp Rev Neurother. 2015;15(3):251-68.

22. Oh J, O'Connor PW. Teriflunomide in the treatment of multiple sclerosis: current evidence and future prospects. Ther Adv Neurol Disord. 2014;7(5):239-52.

23. Kieseier BC, Benamor M. Pregnancy outcomes following maternal and paternal exposure to teriflunomide during treatment for relapsing-remitting multiple sclerosis. Neurol Ther. 2014;3(2):133-8. 